\title{
Modeling and Synthesis of Tactile Texture with Spatial Spectrograms for Display on Variable Friction Surfaces
}

\author{
David J. Meyer, Michael A. Peshkin, and J. Edward Colgate
}

\begin{abstract}
Texture modeling strives to encapsulate the important properties of texture in a concise representation for interpretation, storage, and rendering. Models for tactile texture have yet to describe a representation that is both perceptually complete and sufficiently compact. In this work, we take inspiration from models of visual and auditory texture and propose a spatial spectrogram representation of tactile texture that separates localized features from textural aspects using a windowed Fourier decomposition. We investigate the length scales at which humans can perceive localized features, and represent textures as spectrograms that capture those local features. Additionally, we demonstrate a reconstruction algorithm capable of recreating texture from a spectrogram representation with no perceptual consequence.
\end{abstract}

\section{INTRODUCTION}

The successful digitization of auditory and visual information is in evidence every time music or a video plays on an electronic device. In contrast, while the tactile modality provides another important pathway for information, the digitization, transmission and replay of this information remains primitive. In order for this to change, more advanced display technologies and digitization techniques are both needed. With regard to display, recent developments in surface haptics would appear to provide a method for displaying rich tactile textures and shapes in a digitized, programmable fashion. This paper addresses the digitization challenge, specifically with regard to tactile textures.

To enable the digitization of textures, it is crucial to have an understanding of how they are perceived, and to develop a model for representing what is perceived. A texture model comprises a set of functions that compute statistical parameters representing the texture. We seek a texture model that computes parameters such that textures co-located in the parameter space are perceptually equivalent. Furthermore, it is desired that any two textures are perceptually distinguishable if and only if they exhibit different parameters as determined by the model. The development of a texture model with the aforementioned characteristics will both provide insight to tactile perception and facilitate synthesis of texture for haptic display.

\section{BACKGROUND}

Much of what is currently known about texture comes from studies of preattentive visual perception [1]. In this field, a successful approach has been to employ banks of

DJ Meyer, MA Peshkin, and JE Colgate are with the Department of Mechanical Engineering, Northwestern University, 2145 N Sheridan Rd, Evanston, IL 60208, USA E-mail: meyerdj@u.northwestern.edu; peshkin@northwestern.edu; colgate@northwestern.edu length-scale and orientation specific spatial filters to represent the first stage of texture discrimination [2], [3]. Statistics taken on the outputs of these filter banks have been used to synthesize textures that, in early visual perception, are indiscriminable from the input textures [4]. Similarly, analyses of sound textures have typically employed banks of bandpass filters that mimic the filtering done by the cochlea[5]. The resulting representation of sound data has been separated into two different types of information: the slowly varying envelopes of the band-passed filtered signals, and the rapidly varying fine details of the sound. Several studies have shown the usefulness of representing sound texture signals in this manner, both for understanding perceptual information and for measuring and synthesizing natural sound textures [6]. Slowly varying envelopes were discovered to be important in the perception and interpretation of speech, while the high-frequency fine details of sound were more important for sound location [7]. Combining the statistics from both the time-varying envelopes and the finer spectral content has been shown to produce higher-fidelity reproductions of sound than using spectral content alone [8], [9].

Haptics researchers have also proposed parameter-based models of texture for synthesis and discrimination, but standard approaches have been slow to emerge, perhaps because there is no universally accepted method for tactile texture display and measurement, as exists for sound and vision. Multi-dimensional scaling (MDS) techniques have suggested that natural tactile textures can fall into three or four orthogonal dimensions: roughness, hardness, coldness, and potentially stickiness [10], [11]. Unfortunately, MDS techniques do not model texture in a way that is useful for synthesis or computational storage, as the technique obtains data by polling the human perception directly. A few studies have combined MDS techniques with adjective-scale ratings and physical measurements of textured surfaces [12], [13].

Alternatively, some modeling techniques that analyze the vibratory signatures of textures have been proposed. Spatial spectra [14] and temporal spectra as a function of normal force and velocity of contact have been rigorously studied in efforts to perform texture discrimination [15], [16] as well as texture synthesis in tool-based interactions [17]. While these studies have shown the ability to make predictions about tactile perception, they have not yet led to a computationally efficient method of texture representation and synthesis with demonstrated perceptual relevance.

The human body perceives tactile texture in a much more complex way than vision or sound. Visual textures are signals in two-dimensional space only, and sound textures are signals 
in time only. Because visual channels are limited in bandwidth and spatially localized, Gabor filters are well-suited to serve as basis functions for visual texture representation. Similarly, since the cochlea effectively decomposes sound into a series of different frequency bands, band-pass filters and their respective amplitude envelopes are an appropriate first processing step for sound texture modeling. Tactile perception, on the other hand, is mediated by a collection of neural receptors that detect spatial variations and temporal variations. The contributions of three different types of mechanoreceptors in the skin allow for complex signal processing of mechanically derived signals as a fingertip scans over a texture [18]. Because of this complexity, it is not trivial to assign a set of basis functions for a model.

A simplified yet still elusive problem is that of modeling texture perception on a surface haptic device. Friction modulation surface haptic devices, such as TPad [19], [20], have only one degree of freedom for actuation; they can simply display a variable level of friction between the fingertip and surface. Because of this limitation, true spatial information is not presented to the fingertip. Therefore, the major tactile perception pathway for surface haptics would appear to be the Pacinian channel, which encodes high-frequency vibrations as the finger scans across a textured surface. Since the Pacinian corpuscles are very poor at resolving spatial information [21], the texture signal is perceived as a function of time. Motivated by the use of short-time Fourier transform-like analyses in both vision and sound, we propose a method for texture representation that distinguishes localized features from spectral vibrations, via a windowed Fourier decomposition that creates a spatial spectrogram.

The main parameters in a windowed Fourier decomposition are the size of the window and the spatial resolution of the sampled windows. The size of the window dictates the resolution and range of the spatial-frequency domain represented by the model. A wider window provides finer resolution in the spatial-frequency domain, which is desirable for making texture distinctions. However, for a given window overlap, a wider window decreases the spatial localization accuracy of the representation, which negatively affects representation of localized features. In this work, we investigate the length scale at which localization accuracy is important for early tactile perception.

\section{FEATURE LocAlizATION}

\section{A. Haptic Display}

The friction modulation device used in this work is a TPad that employs ultrasonic vibration to reduce surface friction. Because there is only one actuator, two touch points cannot be separately friction-controlled, and therefore, all interaction is limited to a single touch point only. The rendering of texture on the surface is achieved by controlling the friction based on the state of the fingertip in contact. Relevant state variables include touch position and velocity, and possibly touch pressure, although we did not make use of touch pressure for the work presented here.
To study texture perception effectively, the haptic actuator must be able to render textures in a way that exceeds the human discrimination thresholds. In the case of friction modulation, this is achieved by building a device that can modulate friction with a wide temporal bandwidth. The device developed for these experiments has been presented in previous publications and is shown to perform well over the range of frequencies relevant to tactile perception [22], [23]. Friction values are commanded at an $8.33 \mathrm{kHz}$ rate. The touch point location is measured at the same $8.33 \mathrm{kHz}$ rate using a custom single-axis optical sensor that provides a resolution of 5.3 microns. For the purposes of this research, the friction level varies only as a function of touch location.

\section{B. Test Textures}

We designed a set of textures to test participants' ability to detect spatially localized events. The textures are formed from a 1/f noise signal band-limited between $5.0 \mathrm{~mm}$ and 25 microns in spatial wavelength. A square wave of varying wavelength is used in full amplitude modulation to create 'gaps' in the noisy texture. Wide gaps in the texture (e.g. $5 \mathrm{~mm}$ ) are easy to perceive as localized features on the display. Small gaps (e.g. $0.1 \mathrm{~mm}$ ) are difficult to detect as they are felt as part of the texture. We measured participants' ability to detect these gaps as a function of gap size. As a distractor, we formed a texture having the same spectral content as the gap-containing texture, but with randomized phase. Shown in figure 1 are two such combinations of modulated texture and its distractor, side-by-side.

We hypothesize a threshold gap size below which the phase of the texture signal is not detected, and above which the finger localizes the gaps on the surface. It is this distinction between localized features and textural features that the windowed Fourier decomposition extracts and presents in two dimensions. The hypothetical gap width is important for a decomposition that captures perceptually relevant information, as can be seen in figure 1 in the spatial spectrograms of the designed textures. The spectrograms created with a window of $2 \mathrm{~mm}$ show hardly any distinction between the $1 \mathrm{~mm}$ modulated texture and its distractor, whereas the $0.2 \mathrm{~mm}$ window decomposition makes obvious the spatial distribution of spectral energy. We seek a window width for the decomposition such that the spectrogram distinguishes between textures that feel different, but not between those that feel similar.

\section{Experiment 1: Gap Detection}

To determine the gap size threshold, we designed a twointerval, forced-choice, one-up, four-down adaptive procedure. For each trial, a texture created as shown in figure 1 was displayed. The subject was asked to report the side of the display on which the gaps in the texture were rendered: left or right. The procedure began with a gap width of 6 $\mathrm{mm}$ and decreased upon four consecutive correct answers and increased upon one incorrect answer. The width was changed by a factor of 16,8,4 and 2 on the first four reversals respectively to speed up the rate of convergence. After the 


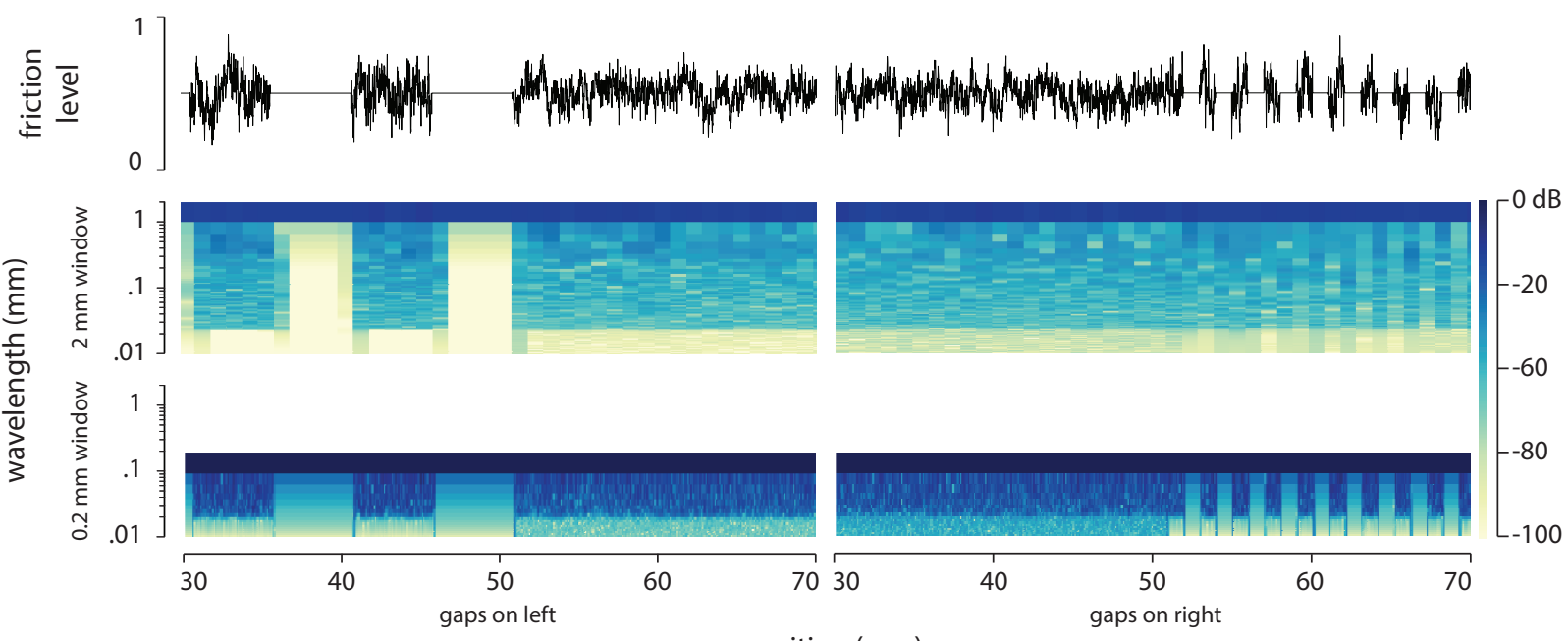

position (mm)

Fig. 1. Left: a modulated texture with $5 \mathrm{~mm}$ gaps and its distractor. Right: a texture with $1 \mathrm{~mm}$ gaps and its distractor. The spectrograms are deconstructed forms of the texture with window widths of $2 \mathrm{~mm}$ (middle) and $0.2 \mathrm{~mm}$ (bottom). The $2 \mathrm{~mm}$ spectrogram distinguishes $5 \mathrm{~mm}$ features from the distractor, but does not represent $1 \mathrm{~mm}$ features as separate from the distractor. The $0.2 \mathrm{~mm}$ spectrogram distinguishes both size features.

initial four reversals, the factor was calculated as $1+1 / \mathrm{R}$, where $\mathrm{R}$ is the reversal number. The gap widths of the final 6 reversals were averaged to obtain the detection threshold.

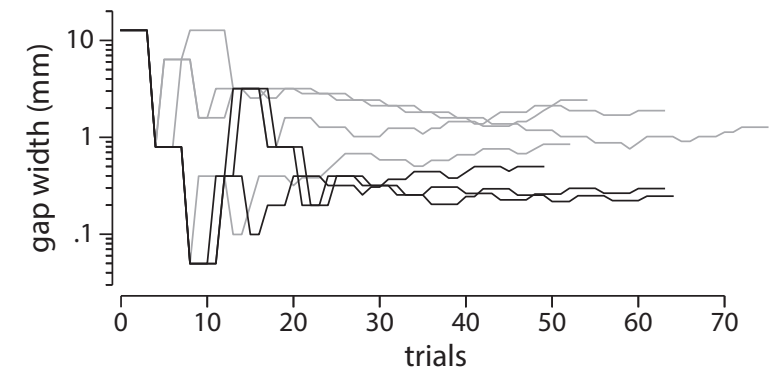

Fig. 2. Seven participants converged to detecting gap sizes of $0.2 \mathrm{~mm}$ to $2 \mathrm{~mm}$, with an average of $0.96 \mathrm{~mm}$. Three expert participants are shown in dark color.

A touchscreen tablet placed on the table next to the TPad provided written instructions, asked questions, and received answers from the participant during the experiment. At the start of a trial, the participant was instructed via the tablet to feel the texture. One half-second later, to allow for the subject to react, the texture was displayed on the TPad, with gaps placed randomly on the left or right. Because we are interested in a spectrogram representation that is independent of exploratory procedure, participants were instructed to explore the texture with any method they chose. After two seconds the texture disappeared and the subject was asked to identify the side of the screen on which the gaps were presented: left or right. To facilitate faster learning, feedback regarding correct or incorrect responses was given during the first two reversals. A texture visual similar to figure 1 was shown and explained to the participants before the start of the experiment. The procedure was reviewed by the Northwestern University Institutional Review Board and all participants gave informed consent before participating.

\section{Results}

Seven participants' data are shown in figure 2. We observe a wide range of thresholds, from $0.25 \mathrm{~mm}$ to $2 \mathrm{~mm}$ with an average of $0.96 \mathrm{~mm}$. An eighth subject could not perceive the texture gaps even at the widest possible rendering, and is therefore not shown. Three participants stood out as experts, characterized by their familiarity with the texture display and stable threshold values; their data is shown in the dark lines. These participants showed the ability to distinguish between the gapped textures and the distractors down to gap widths of 250 microns.

The purpose of this experiment was to discover the length scales at which participants no longer felt spatial features and instead felt textural features. The results suggest that at distances shorter than 250 microns, phase information is not relevant to early texture perception. The spatial spectrogram is therefore a perceptually relevant representation of texture, because it discards phase information at high spatial frequencies.

\section{Texture ReCONSTRUCtion}

\section{A. Algorithm}

An ideal texture model not only provides a simplified parameter space, but also a method for synthesizing texture from those parameters. As we investigate the spatial spectrogram as a first-step analysis for texture representation, we must also take note of the ability to construct textures from the information in the spectrogram. We developed an algorithm that uses the phase information stored in the DC value of the Fourier transform at each window location to initialize the phase for the inverse Fourier transform. For each point in space, the friction value is a sum of the points calculated from two overlapping windows in the spectrogram. 


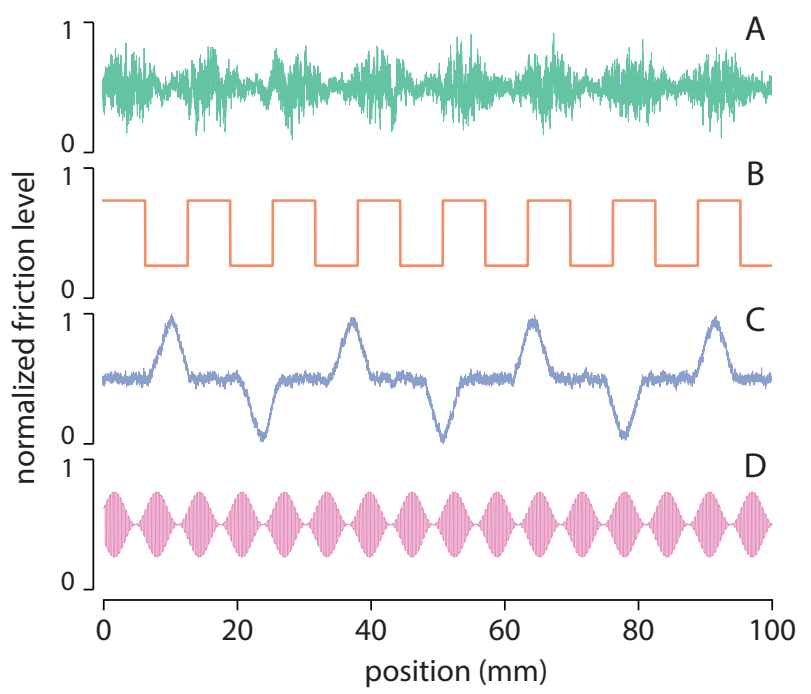

Fig. 3. Texture $\mathrm{A}$ is the $1 / \mathrm{f}$ noise signal $70 \%$ amplitude modulated with a $13 \mathrm{~mm}$ wavelength sine wave. Texture B is a $13 \mathrm{~mm}$ square wave, Texture C is a series of large features with added $1 / \mathrm{f}$ noise, and Texture $\mathrm{D}$ is a $0.4 \mathrm{~mm}$ square wave, $100 \%$ amplitude modulated by a $6 \mathrm{~mm}$ sine wave.
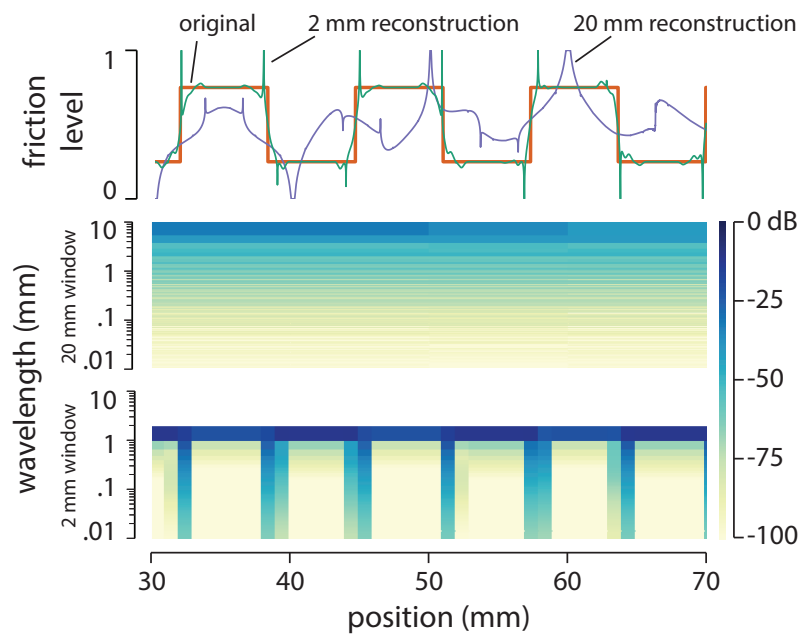

Fig. 4. Texture B, a pure square wave, is shown along with its reconstructions from the two spectrograms shown. The spectrograms were created with window widths of $20 \mathrm{~mm}$ (middle) and $2 \mathrm{~mm}$ (bottom).

To validate the reconstruction and evaluate various window sizes, we conducted an experiment using four heuristically designed textures, shown in figure 3. These textures were designed to incorporate small and large natural and synthetic properties. Each texture was deconstructed into a spatial spectrogram and then reconstructed using 7 different window widths, varying from $20 \mathrm{~mm}$ to $0.2 \mathrm{~mm}$. The square wave texture with spatial wavelength of about $13 \mathrm{~mm}$ is shown in figure 4 with its spectrograms and reconstructions for window widths of $20 \mathrm{~mm}$ and $2 \mathrm{~mm}$.

\section{B. Experiment 2: Reconstruction Fidelity}

The three expert participants from Experiment 1 were asked to return to evaluate the perceptual difference between the original and the reconstruction for each of the 28 com-

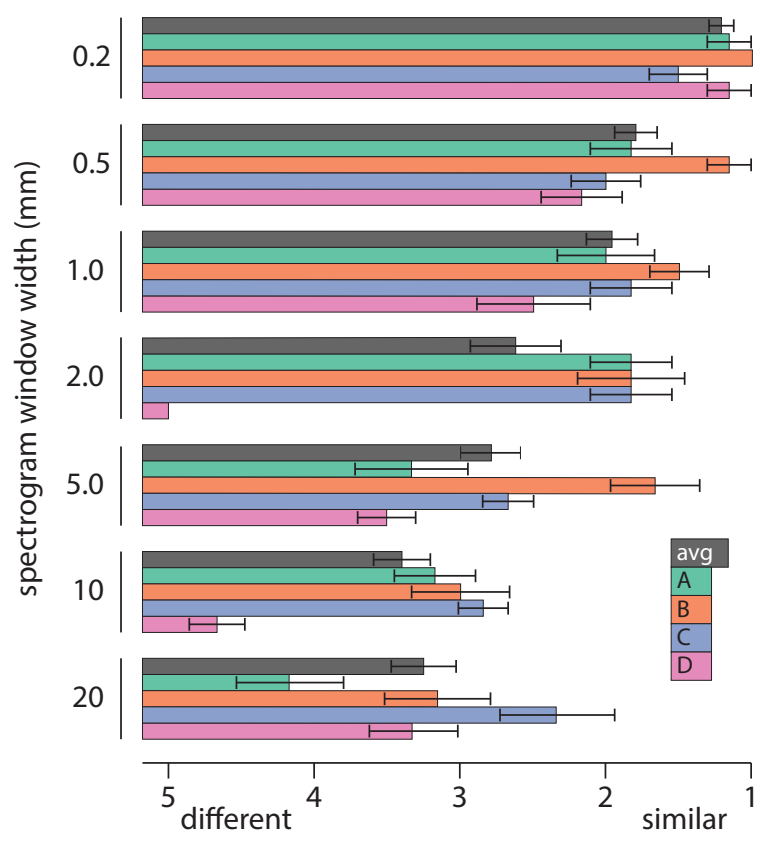

Fig. 5. Similarity ratings between textures and their spectrogram reconstruction. The average of all textures tested is shown in gray, each texture is represented by its color. A similarity rating of 1 was described as indistinguishable by every subject.

binations. For each trial, the subject was presented with a control interface on the tablet to switch between the designed texture and its reconstruction, although they were unaware of which texture was the reconstruction. The subject was free to explore each texture with an exploratory procedure for as long as desired and could switch back and forth as necessary. When the subject felt comfortable with an answer, he/she was asked to rate the difference between the two textures on a scale of 1 to 5,1 being "very similar" and 5 being "very different." The first 24 trials consisted of reconstructions that were spaced across the window size range to provide a reference for the participants. These results were not used in the analysis. In the following 56 trials each of the 28 comparisons was presented twice, all in a random order. As with Experiment 1, the procedure was reviewed by the Northwestern University Institutional Review Board and all participants gave informed consent before participating.

\section{Experiment 2 Results}

All three participants data is shown averaged together in figure 5. Each colored bar represents the data for the respective texture, and the gray bar represents the average of all the textures. Data further to the right indicate a higher fidelity reconstruction from spectrogram. There is a clear trend showing decreasing fidelity as window width increases, although the point at which the reconstruction breaks down differs between textures. At a window width of $0.2 \mathrm{~mm}$, the data show a very strong indication of a rating of 1 . All three participants reported that a response of 1 indicated that they could not distinguish between the two textures. 


\section{Discussion}

Participant performance in Experiment 1 makes it clear that some individuals familiar with feeling surface haptic devices are highly attuned to subtle texture differences. Discussion with these participants suggests that this is not just a matter of sensory acuity, but that they are more experienced with varying exploratory procedures to glean tactile information from the display. Indeed, as the adaptive procedure rendered smaller size gaps, the expert participatns reported they were no longer feeling distinct gaps in the texture, but only a slightly more rough feeling. This could be an indication that non-uniformity of the distribution of spectral energy in space contributes to the perception of roughness. Such a result would be consistent with established models of roughness perception [24], [25], although those models are based on SAI afferent responses, which are unlikely to be significant contributors in the case of the TPad due to its extremely flat surface.

The fact that experts were cued by roughness instead of gap detection at the small gap sizes is of interest. At sufficiently small wavelengths, participants cannot feel localized features, but phase information is apparently still relevant. For a spectrogram, the phase information associated with spectral content smaller than the window width is discarded; thus, the only way to capture all distinctions between textures is with a rather narrow window width, as found in Experiment 1. However, other texture models may capture roughness (as well as other qualitative aspects of texture) in a different way, in which case their parameters may not need to vary with such a fine spatial resolution.

The reconstruction of textures from the spectrogram representation demonstrates that it has the ability to store perceptually relevant information. All participants reported that a response of 1 indicated no perceptual difference between textures, and that a response of 5 indicated immediate and obvious distinction. It should be appreciated that this experiment compared reconstructed textures with only their own original designs. Had the reconstructions been used in an identification task, or in comparisons with other textures, it is very likely they would have been rated very similar for window widths of $1 \mathrm{~mm}$ and lower. At a $2 \mathrm{~mm}$ wavelength, the reconstruction evidently breaks down for one of textures, but not for others.

A wider window for spectrogram reconstruction indicates a greater possibility for data compression. While these spectrograms were not data compressed, our goal is to represent the spectral content with a set of parameters that greatly reduces the data storage necessary to recreate the texture. With a window width of $1 \mathrm{~mm}$, spectral data need to be stored only once for each $0.5 \mathrm{~mm}$ of space, and wider windows lead to even fewer data points. It is interesting to observe that certain textures are more susceptible to degradation with increasing window width than others. On the basis of these four textures only, it seems that textures with larger features can withstand further compression before perception is affected. It may be possible to calculate a statistic on the texture to predict the widest window that can capture all perceptual relevance in order to optimize a data compression algorithm.

\section{CONCLUSION}

In this paper, we have demonstrated that spatial spectrograms may be used to represent texture waveforms for friction-modulating surface haptic devices. We experimentally determined the smallest feature size participants could locate during early texture perception, and found $0.25 \mathrm{~mm}$ to be a conservative estimate of this value. Additionally, we reconstructed textures from spectrograms of a set of pre-designed textures. We tested the similarity between the reconstructed textures and the original textures, finding that with a window size of $0.2 \mathrm{~mm}$, the reconstructions are indistinguishable from their respective originals. Although not developed here, it also seems likely that the spatial spectrogram will enable the compression of texture data. It is important to understand, however, that this work has not addressed the realism of virtual textures as compared to any physical counterparts, nor have the models taken into account other variables of fingertip contact, such as velocity and contact force. In light of these factors, it seems likely that more sophisticated texture representation and reconstruction techniques will ultimately be necessary.

\section{APPENDIX}

\section{A. Spectrogram Decomposition}

Given a texture, $f$, as a function of space $x$, one location on the spectrogram is calculated by a discrete Fourier transform with an $N$-wide Hanning window function. The spectrogram is calculated at locations in space separated by half the window width such that each window overlaps with its two neighbors only. The function to calculate the spectrogram is:

$$
\begin{gathered}
S\left(x_{m}, k\right)=\left.|| \sum_{n=0}^{N-1} w(n) f\left(x_{m+n-\frac{N}{2}}\right) e^{-i 2 \pi k \frac{n}{N}}\right|^{2} \\
\text { for } \quad k=0,1, \ldots \frac{N}{2} \\
\quad m \in \mathbb{M}, \mathbb{M}=\left\{\frac{N}{2}\left(1,2, \ldots \frac{2 L}{N}-1\right)\right\}
\end{gathered}
$$

where $\mathrm{S}$ is the function of space $(x)$ and spatial frequency represented by normalized wave number $(k)$. The window function is represented by $w$ and is centered at $w\left(\frac{N}{2}\right) . L$ is the number of points in the texture $f(x)$. The upper half of the standard discrete Fourier transform is discarded as this information is redundant given real input.

\section{B. Texture Reconstruction}

The first step in reconstructing the texture is forming the overall shape from the local DC values in the spectrogram. This is just a sum of the DC values of the neighboring spectrogram data. The initial shape is calculated as follows

$$
\begin{aligned}
& d_{n}=\frac{1}{N} \sum_{m \in \mathbb{A}_{n}} \sqrt{S\left(x_{m}, 0\right)}, \quad \text { for } n=0,1 \ldots L-1 \\
& \quad \mathbb{A}_{n} \subset \mathbb{M}=\forall m\left\{m \in \mathbb{M},-\frac{N}{2} \leq(n-m)<\frac{N}{2}\right\}
\end{aligned}
$$


where $d$ is the value at all points $n$ in space and $\mathbb{A}_{n}$ is the set of indexes of the spectrogram whose window encloses point $n$. The overall shape is used to calculate phase values to be used in the inverse transform as follows:

$$
\begin{gathered}
P\left(x_{m}, k\right)=\angle\left(\sum_{n=0}^{N-1} w(n) d\left(x_{m+n-\frac{N}{2}}\right) e^{-i 2 \pi k \frac{n}{N}}\right) \\
\text { for } \begin{array}{l}
k=0,1, \ldots \frac{N}{2} \\
m \in \mathbb{M}
\end{array} \\
F\left(x_{m}, k\right)=\sqrt{S\left(x_{m}, k\right)} e^{i P\left(x_{m}, k\right)}
\end{gathered}
$$

where $\mathrm{P}$ is only the phase information of the windowed Fourier transform.

The inverse Fourier transforms are performed on $F$, which contains the magnitude information from the spectrogram and the phase information from the newly derived $\mathrm{P}$ matrix. $f\left(x_{n}, m\right)$ derived below represents the texture value at point $x_{n}$ based on the inverse transform from the spectrogram at point $x_{m}$.

$$
\begin{aligned}
f\left(x_{n}, m\right)=\frac{1}{N} & \left(\sum_{k=0}^{N / 2} F\left(x_{m}, k\right) e^{i 2 \pi\left(n-m+\frac{N}{2}\right) \frac{k}{N}}\right. \\
& \left.+\sum_{k=1}^{N / 2-1} F^{*}\left(x_{m}, N-k\right) e^{i 2 \pi\left(n-m+\frac{N}{2}\right) \frac{N-k}{N}}\right)
\end{aligned}
$$

To obtain the final reconstruction value, an overlap-add method is used. $f\left(x_{n}, m\right)$ is windowed and summed over all non-zero values $\left(\forall m \in \mathbb{M}_{n}\right)$ as follows:

$$
f\left(x_{n}\right)=\frac{\sum_{m \in \mathbb{M}_{n}} f\left(x_{n}, m\right) w\left(n-m+\frac{N}{2}\right)}{\sum_{m \in \mathbb{M}_{n}} w^{2}\left(n-m+\frac{N}{2}\right)}
$$

\section{ACKNOWLEDGMENT}

This material is based upon work supported by the National Science Foundation under Grants No. IIS-1302422 and IIS-0964075.

\section{REFERENCES}

[1] B. Julesz and J. Bergen, "Human factors and behavioral science: Textons, the fundamental elements in preattentive vision and perception of textures," Bell System Technical Journal, The, vol. 62, no. 6, pp. 1619-1645, July 1983.

[2] A. Jain and F. Farrokhnia, "Unsupervised texture segmentation using gabor filters," in , IEEE International Conference on Systems, Man and Cybernetics, 1990. Conference Proceedings, Nov. 1990, pp. 14-19.

[3] A. Sutter, G. Sperling, and C. Chubb, "Measuring the spatial frequency selectivity of second-order texture mechanisms," Vision Research, vol. 35, no. 7, pp. 915-924, Apr. 1995.

[4] J. Portilla and E. P. Simoncelli, "A parametric texture model based on joint statistics of complex wavelet coefficients," International Journal of Computer Vision, vol. 40, no. 1, pp. 49-70, Oct. 2000.

[5] B. Gygi, G. R. Kidd, and C. S. Watson, "Spectral-temporal factors in the identification of environmental sounds," The Journal of the Acoustical Society of America, vol. 115, no. 3, pp. 1252-1265, Mar. 2004.

[6] J. M. Grey and J. W. Gordon, "Perceptual effects of spectral modifications on musical timbres," The Journal of the Acoustical Society of America, vol. 63, no. 5, pp. 1493-1500, May 1978.

[7] Z. M. Smith, B. Delgutte, and A. J. Oxenham, "Chimaeric sounds reveal dichotomies in auditory perception," Nature, vol. 416, no. 6876, pp. 87-90, Mar. 2002.
[8] X. Serra, "Spectral modeling synthesis: A sound analysis/synthesis based on a deterministic plus stochastic decomposition," Computer Music Journal, vol. 14, pp. 12-24, 1990.

[9] J. H. McDermott and E. P. Simoncelli, "Sound texture perception via statistics of the auditory periphery: Evidence from sound synthesis," Neuron, vol. 71, no. 5, pp. 926-940, Sept. 2011.

[10] M. Hollins, R. Faldowski, S. Rao, and F. Young, "Perceptual dimensions of tactile surface texture: A multidimensional scaling analysis," Perception \& Psychophysics, vol. 54, no. 6, pp. 697-705, Nov. 1993.

[11] S. Okamoto, H. Nagano, and Y. Yamada, "Psychophysical dimensions of tactile perception of textures," IEEE Transactions on Haptics, vol. 6, no. 1, pp. 81-93, 2013.

[12] W. M. Bergmann-Tiest and A. M. L. Kappers, "Analysis of haptic perception of materials by multidimensional scaling and physical measurements of roughness and compressibility," Acta Psychologica, vol. 121, no. 1, pp. 1-20, 2006.

[13] T. Yoshioka, S. Bensmaia, J. Craig, and S. Hsiao, “Texture perception through direct and indirect touch: An analysis of perceptual space for tactile textures in two modes of exploration," Somatosensory \& motor research, vol. 24, no. 1-2, pp. 53-70, 2007.

[14] M. Wiertlewski, J. Lozada, and V. Hayward, "The spatial spectrum of tangential skin displacement can encode tactual texture," IEEE Transactions on Robotics, vol. 27, no. 3, pp. 461-472, 2011.

[15] D. Xu, G. Loeb, and J. Fishel, "Tactile identification of objects using bayesian exploration," in 2013 IEEE International Conference on Robotics and Automation (ICRA), May 2013, pp. 3056-3061.

[16] J. A. Fishel and G. E. Loeb, "Bayesian exploration for intelligent identification of textures," Frontiers in Neurorobotics, vol. 6, June 2012.

[17] H. Culbertson, J. Unwin, B. Goodman, and K. Kuchenbecker, "Generating haptic texture models from unconstrained tool-surface interactions," in World Haptics Conference (WHC), 2013, Apr. 2013, pp. 295-300.

[18] A. I. Weber, H. P. Saal, J. D. Lieber, J.-W. Cheng, L. R. Manfredi, J. F. Dammann, and S. J. Bensmaia, "Spatial and temporal codes mediate the tactile perception of natural textures," Proceedings of the National Academy of Sciences, vol. 110, no. 42, pp. 17 107-17 112, Oct. 2013

[19] L. Winfield, J. Glassmire, J. E. Colgate, and M. Peshkin, "T-PaD: Tactile pattern display through variable friction reduction," in EuroHaptics Conference, 2007 and Symposium on Haptic Interfaces for Virtual Environment and Teleoperator Systems. World Haptics 2007. Second Joint, Mar. 2007, pp. 421 -426.

[20] N. Marchuk, J. Colgate, and M. Peshkin, "Friction measurements on a large area TPaD," in Haptics Symposium, 2010 IEEE, Mar. 2010, pp. $317-320$

[21] S. J. Bensmaia, "Tactile intensity and population codes," Behavioural Brain Research, vol. 190, no. 2, pp. 165-173, July 2008.

[22] D. Meyer, M. Wiertlewski, M. Peshkin, and J. Colgate, "Dynamics of ultrasonic and electrostatic friction modulation for rendering texture on haptic surfaces," in 2014 IEEE Haptics Symposium (HAPTICS), Feb. 2014, pp. 63-67.

[23] M. Wiertlewski, D. Leonardis, D. J. Meyer, M. A. Peshkin, and J. E. Colgate, "A high-fidelity surface-haptic device for texture rendering on bare finger," in Haptics: Neuroscience, Devices, Modeling, and Applications, ser. Lecture Notes in Computer Science, M. Auvray and C. Duriez, Eds. Springer Berlin Heidelberg, June 2014, pp. 241-248.

[24] C. E. Connor, S. S. Hsiao, J. R. Phillips, and K. O. Johnson, "Tactile roughness: neural codes that account for psychophysical magnitude estimates," The Journal of Neuroscience, vol. 10, no. 12, pp. 38233836, Dec. 1990.

[25] C. E. Connor and K. O. Johnson, "Neural coding of tactile texture: comparison of spatial and temporal mechanisms for roughness perception," The Journal of Neuroscience, vol. 12, no. 9, pp. 3414-3426, Sept. 1992. 